\section{Circadian effects on stroke outcome - Did we not wake up in time for neuroprotection?}

\author{
Johannes Boltze', Nadine Didwischus', Martha Merrow², \\ Robert Dallmann ${ }^{3}$ and Nikolaus Plesnila ${ }^{4,5}$ \\ Robert Dallmann and Nikolaus Plesnila
}

Journal of Cerebral Blood Flow \& Metabolism

202I, Vol. 4I (3) 684-686

(C) The Author(s) 2020

\section{(c) (i) (8)}

Article reuse guidelines: sagepub.com/journals-permissions DOI: |0. I |77/027|678X209787 | | journals.sagepub.com/home/jcbfm @SAGE

\begin{abstract}
The occurrence of stroke in humans peaks in the morning. A recent study revealed that time of day mitigates the therapeutic impact of neuroprotective paradigms. These findings might not only explain the previous failure of translation of neuroprotective therapies but inspire new paradigms in stroke chronopathophysiology research. Taking chronotype into account may complement the many factors that influence efficacy of experimental therapies in stroke.
\end{abstract}

\title{
Keywords
}

Stroke, animal models, neuroprotection, chronobiology. translational research

Received 2I July 2020; Revised 23 September 2020; Accepted 25 September 2020

Over the past two decades, numerous reasons have been discussed for the failure of classical neuroprotective paradigms in ischemic stroke to translate successfully to clinical practice. Many of those reasons relate to the predictive value of stroke models as well as preclinical study design limitations such as improper randomization and blinding strategies. Researchers learnt quickly from previous translational setbacks. Major improvements in methodological quality were achieved in the stroke field, which may now have outpaced other areas of cardiovascular research in this regard. ${ }^{1}$ Moreover, an increasing body of evidence suggests that animal models adequately reflect human stroke pathophysiology and outcome when used appropriately. Despite this, translational research on neuroprotectants remained futile. This refocused our attention from pretended fundamental limitations in preclinical research models and strategies to seemingly minor yet impactful design differences between preclinical and clinical studies.

Recently, a potentially game-changing study revealed a surprising influence of treatment time on stroke outcome, ${ }^{2}$ adding to translationally relevant study design differences. ${ }^{3}$ A number of established neuroprotective paradigms (normobaric hyperoxia, radical scavenging, NMDA receptor antagonism) were evaluated for efficacy during the inactive (light phase) versus the active (dark phase) phase in nocturnal rodents after stroke. Major treatment effects were only seen for interventions during the inactive phase. A major reason for the phenomenon was a significantly smaller penumbra during the active phase. These findings are highly relevant and timely given the supposed renaissance of neuroprotective approaches in the reperfusion era. ${ }^{4}$ Indeed, treatments in clinical trials are predominantly performed during the day-time (human active phase) for logistical reasons. If temporally regulated

\footnotetext{
'School of Life Sciences, University of Warwick, Coventry, UK

${ }^{2}$ Faculty of Medicine, Institute of Medical Psychology, LMU Munich, Munich, Germany

${ }^{3}$ Division of Biomedical Sciences, Warwick Medical School, University of Warwick, Coventry, UK

${ }^{4}$ Institute for Stroke and Dementia Research (ISD), Munich University Hospital and Faculty of Medicine, LMU Munich, Munich, Germany

${ }^{5}$ Munich Cluster of Systems Neurology (Synergy), Munich, Germany

Corresponding author:

Johannes Boltze, School of Life Sciences, University of Warwick, Coventry CV4 7AL, UK.

Email: johannes.boltze@warwick.ac.uk
} 
physiology plays a role in the observed neuroprotective effects, this may partially explain the translational failure of neuroprotective treatments we were experiencing.

Although novel and striking, these findings should not come as a major surprise. Many biological functions relevant for stroke pathophysiology are orchestrated by the circadian clock (Figure 1) and may thus be of relevance for therapeutic interventions. For instance, blood pressure shows a clear diurnal profile reaching its nadir at the end of the resting period. In turn, susceptibility to antihypertensive interventions varies throughout the day. Cerebral blood vessels undergo diurnal changes in vascular function that are relevant to pathology. ${ }^{5}$ Moreover, there is a circadian rhythm in leukocyte migration and function, including immune reactions. ${ }^{6}$ Leukocytes egress the circulation and accumulate in tissues with a marked daily, temporal program. Leukocyte accumulation is mediated by increased adhesion molecule expression on endothelial cells during the active phase, and is proposed to contribute to worse outcome after myocardical infarction occurring in the wake phase versus the resting phase. This could also be of direct relevance to secondary neuroinflammatory damage and outcome after ischemic stroke. Finally, the circadian clock is believed to have an influence on redox homeostasis and thereby on neuronal antioxidative defense ${ }^{7}$ that is important for recanalization therapies and research on reperfusion injury.
What are the implications of these findings for future translational research? First, validation of time of day effects in diurnal model systems should be performed. Large animals may help with this as they are mostly diurnal and better reflect human brain anatomy and physiology. Large animal stroke models are increasingly used for assessing reperfusion therapies and are becoming more widely available, ${ }^{8}$ paving the way for tandem reperfusion and neuroprotection studies.

Second, the relevance of circadian regulation for other experimental treatment strategies should be elucidated. For instance, pre- and postconditioning strategies are currently assessed as powerful supplementary therapies that may significantly improve stroke outcome. ${ }^{9}$ Since such strategies predominantly utilize changes in local and systemic metabolic processes that are in turn modulated by circadian clocks, the effect of intervention time should be systemically investigated. Moreover, the impact of circadian rhythms should be considered when optimizing strategies measuring functional outcome that may reveal different results in the active versus the inactive phase.

Deciphering time of day effects is time-consuming. A third, interim suggestion would be to alter current experimental practices when nocturnal species such as rodents are used. Reversal of the light dark cycle in animal facilities will harmonize the sleep/wake behavior of animals and researchers. The data presented in Esposito et al. ${ }^{2}$ suggest that acute treatment of stroke

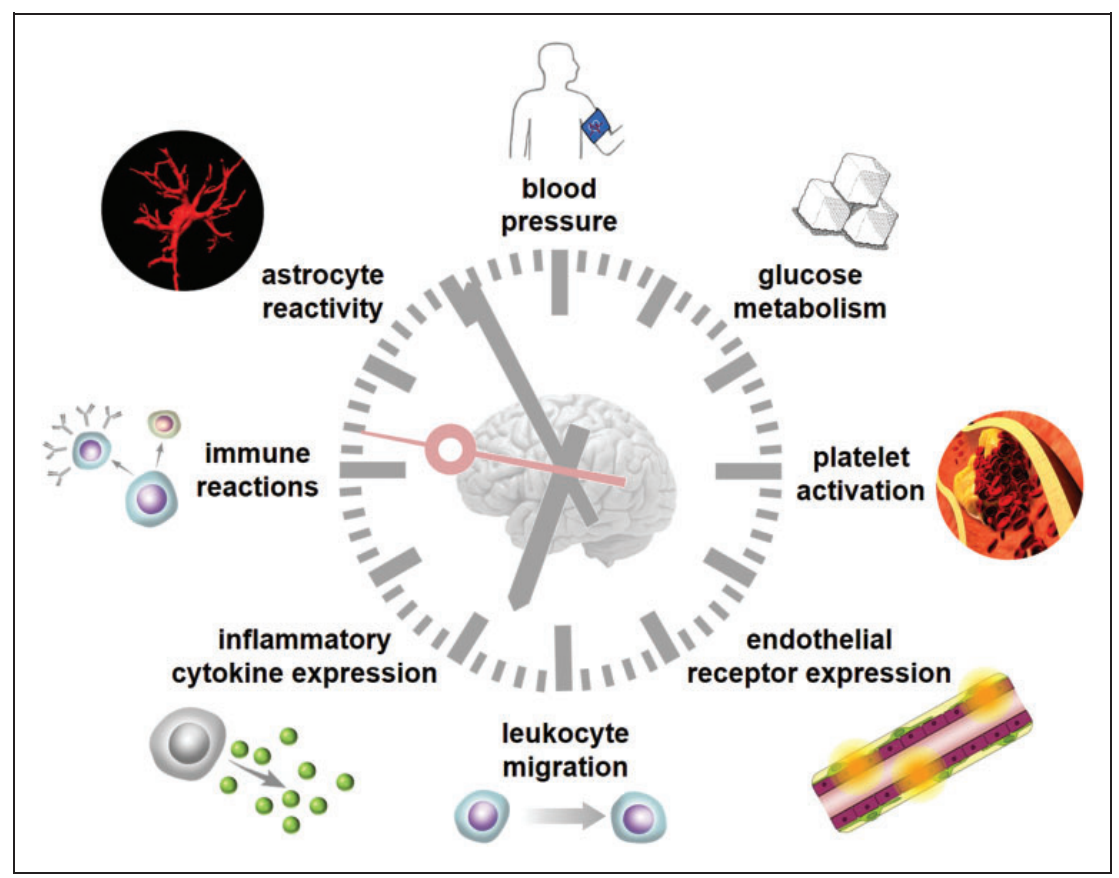

Figure I. Processes relevant to ischemic stroke pathophysiology that are controlled by circadian clocks. 
patients with neuroprotective strategies over the day may not be effective. Thus, if only active (night) times were assayed in previous preclinical experiments, many neuroprotective strategies would have never been discovered. They likely have value but must be implemented appropriately with respect to chronobiological aspects.

Fourth, circadian rhythms are important elements adding to the interplay of factors such as age, sex and comorbidities that can affect stroke outcome and therapeutic efficacy. The sheer number of these factors and therapeutically relevant combinations make it increasingly challenging to model them sufficiently in translational studies. A potential solution may be to more closely interlink preclinical and early-stage clinical studies. Selecting patient populations who reflect the preclinical cohorts in which best effects were seen may increase chances for successful therapeutic intervention. ${ }^{10}$ Selection of eligible patients might be extended beyond criteria such as stroke size or location and may include chronobiological aspects. Stroke patients presenting at night may be assigned to different trials than those presenting during the day.

In summary, the increasing knowledge on circadian clock regulation in stroke and therapeutic outcomes may open the door for new research directions in stroke research. Chrono(patho)physiology might help us to prevent missing out on neuroprotection just by sleeping away the right time for intervention.

\section{Funding}

The author(s) received no financial support for the research, authorship, and/or publication of this article.

\section{Declaration of conflicting interests}

The author(s) declared no potential conflicts of interest with respect to the research, authorship, and/or publication of this article.

\section{References}

1. Ramirez FD, Motazedian P, Jung RG, et al. Methodological rigor in preclinical cardiovascular studies: Targets to enhance reproducibility and promote research translation. Circ Res 2017; 120: 1916-1926.

2. Esposito E, Li W, Mandeville ET, et al. Potential circadian effects on translational failure for neuroprotection. Nature 2020; 582: 395-398.

3. Shi L, Rocha M, Leak RK, et al. A new era for stroke therapy: integrating neurovascular protection with optimal reperfusion. J Cereb Blood Flow Metab 2018; 38: 2073-2091.

4. Marcelo Rocha M, Jadhav AP and Jovin TG. Endovascular therapy for large vessel occlusion stroke: an update on the most recent clinical trials. J Cereb Blood Flow Metab 2019; 39: 1661-1663.

5. Durgan DJ, Crossland RF and Bryan RM Jr. The rat cerebral vasculature exhibits time-of-day-dependent oscillations in circadian clock genes and vascular function that are attenuated following obstructive sleep apnea. J Cereb Blood Flow Metab 2017; 37: 2806-2819.

6. Scheiermann C, Kunisaki Y and Frenette PS. Circadian control of the immune system. Nat Rev Immunol 2013; 13: 190-198.

7. Musiek ES, Lim MM, Yang G, et al. Circadian clock proteins regulate neuronal redox homeostasis and neurodegeneration. $J$ Clin Invest 2013; 123: 5389-5400.

8. Herrmann AM, Meckel S, Gounis MJ, et al. Large animals in neurointerventional research: a systematic review on models, techniques and their application in endovascular procedures for stroke, aneurysms and vascular malformations. J Cereb Blood Flow Metab 2019; 39: 375-394.

9. Zhang J, Zhang W, Gao X, et al. Preconditioning with partial caloric restriction confers long-term protection against grey and white matter injury after transient focal ischemia. J Cereb Blood Flow Metab 2019; 39: 1394-1409.

10. Boltze J, Modo MM, Mays RW, et al.; STEPS 4 Consortium. Stem cells as an emerging paradigm in stroke 4: advancing and accelerating preclinical research. Stroke 2019; 50: 3299-3306. 